\title{
The Application of Three-Dimensional (3-D) Body Scanner in Fabric Drape Assessment
}

\author{
Tannie MAH ${ }^{\mathrm{a}}$ and Guowen SONG* \\ ${ }^{a}$ Consumer Product Safety Directorate, Health Canada, Canada \\ $*^{\mathrm{b}}$ Department of Human Ecology, University of Alberta, Canada
}

\begin{abstract}
In this research three-dimensional (3-D) body scanning technique is applied to assess fabric drape based on air gap information. The objective of the research is to determine whether the air gap information obtained from 3-D scanning can provide a useful and valid measurement of fabric drape, and to investigate if 3-D scanning can produce more information about fabric drape than traditional methods can. The parameters associated with air gap information were examined and the testing protocol to determine the size of air gap was developed. The results demonstrate three-dimensional scanning technique as a means of measuring fabric drape is an advancement over conventional methods. The air gap distance between a fabric and the object over which it is hung can provide information about a draped fabric along its entire length, rather than just a single DC value obtained from a top view. Additionally air gap information can be a more realistic representation of how a garment is draped on a human compared to a single DC value. Considering the average air gap distance in addition to the DC, the number of nodes, and the node dimensions can provide a more complete description of fabric drape than existing methods. This preliminary exploration forms a foundation from which future research using air gap measurements to assess fabric drape can be built.
\end{abstract}

Kewords: 3d body scanning, air gap, drape, DC

\section{Introduction}

Drape refers to a fabric's ability to, and the extent and manner in which, that fabric will fall, flow, or form into folds when hung over an object. Cusick [1] defined drape as a "description of the deformation of the fabric produced by gravity when only part of it is directly supported" (p. T596). Drape describes the final configuration of a fabric when bent under its own weight. The ability to undergo large, recoverable deformations by buckling gracefully into rounded folds of single and double curvature distinguishes fabrics from other sheet materials such as paper [2].

Brand [3] characterized drape as one of six concepts that comprised fabric aesthetic character, as it is an important component influencing the appearance of fabrics. Fabrics have good draping qualities when the sinusoidal folds are pleasing to the eye, suggesting that drape is a qualitative, subjective term [4]. However, drape may also be considered objectively, for example, when thought of as a low-stress physical behaviour influenced by a fabric's stiffness and shear properties, or as a numerical drape coefficient quantifying the amount of drape.

Fabric physical properties also influence drape. The composition (fiber content), structure (yarn type, type of weave, fabric count), and finish of a fabric affect drape. Chu, Platt, and Hamburger [5] found that weaves with longer floats, reduced cover factors, and decreased yarn diameters resulted in higher drape. Knitted fabrics are relatively more drapeable than woven fabrics [6]. The complex mechanisms of drape are increased as a result of the anisotropic (different properties in warp, weft, and bias directions), non-linear, hysteric (history dependent), and visco-elastic characteristics of fabrics [7]. The size, shape, weight, and thickness of a fabric also affect its draping qualities.

Fabric mechanical properties, specifically stiffness (bending and flexural rigidity) and shearing, have been identified as bearing a relationship to drape. Bending is one of the most important mechanical properties when considering curvatures that occur in only one direction, such as in curtains or skirts [8]. The non-resistance of fabrics to bending without external force only under the influence of its true specific weight results in fabric drape [9]. When double curvatures are involved, though, such as in the more complex 3-D drape configurations of garments, bending occurs in more than one direction and shearing comes into play. Shearing occurs when the right-angle orientation of warp and weft yarns are 
shifted as a result of the yarns moving relative to one another [10]. Drape involves large deformations under low stresses, and is affected by a fabric's bending and shear properties [11]. Fabrics with low bending and shear resistances fall into folds more readily [12]. As well, additional variables can affect drape, such as the size and shape of the object over which the fabric is placed, the initial state of the fabric, the effects of humidity, handling procedures, and the time dependence of drape. It is evident that fabric drape is a function of a complex set of optical, physical, mechanical, and other factors

Drape can be judged subjectively and/or objectively, aesthetically and/or mechanically [13]. The subjective evaluation of drape can be performed kinesthetically or visually. In kinesthetic assessment, raters place a hand in a box, pick up and handle a fabric, and determine drape by sensing how the fabric feels when falling around the hand [14]. Subjective methods have therefore generally been deemed unreliable, necessitating the development of more dependable and consistent systems for the measurement of fabric drape [15]. The objective evaluation involves using fabric mechanical properties such as stiffness as a means of indirectly measuring the quality of drape. The methods developed include cantilever method, ring method [8]. However, these methods (2-D tests) are incapable of capturing the anisotropic behavior of fabrics. To overcome the limitations of methods that only gave an indirect indication of the 2-D deformation of fabrics, instruments that could distort a fabric in all three dimensions and provide a direct measure of drape was necessary. Therefore three-dimensional methods represented by a drapemeter were developed by Chu, Cummings, and Teixeira [5] and later improved by Cusick [1]. However all these methods cannot fully capture the truly 3D shape of the fabrics draped of a draped fabric, or garment. With the development of the 3D body scanning technology, it provides a new approach to assess the fabric drape. The current research aims to explore the possibility of using 3-D scanning for fabric drape measurement, and to put forward that the air gap distance is a useful parameter for measuring the drape of fabrics. In this research, 3-D body scanning technology was applied to assess the drape of fabrics based on the information of air-gap size. The parameters associated with air gap information were determined and the testing protocol to measure the size of air gap was developed. The results demonstrate three-dimensional scanning technique as a means of measuring fabric drape is an advancement over conventional methods. This preliminary exploration forms a foundation from which future research using air gap measurements can be built.

\section{Technical approach}

In the current research, a new means of conceptualizing the drape of fabrics is brought forward, in which drape is characterized by the average air gap distance between an object and the textile placed over it. The air gap distance is defined as the distance from the outside edge of a cylinder to the outside edge of a fabric, measured from a point on the cylinder that radiates from the center of the cylinder to the edge of the fabric (Figure 1). The average air gap distance taken from cross-sections at different elevations of a draped fabric (Figure 2) is proposed as a means of assessing the amount of fabric drape.

This is based on the principle that the air gap distance will vary depending on the amount of drape that a fabric exhibits. For example, consider two different fabrics of the same diameter draped over the same cylinder (Figure 3). The fabric on the left is less drapeable than the fabric on the right, as it does not conform to the shape of the cylinder as well. The fabric on the right is more drapeable, as it falls around the cylinder more closely. The fabric on the left has larger air gap distances than the fabric on the right, which has smaller air gap distances.

Seven fabrics (A- G) were selected for drape testing and fabric selection was based on choosing those fabrics that were thought to represent as wide a range of drape values as possible, and were not chosen based on their specific properties. In order to see how well the drape values obtained from the new 3-D scanning approach corresponded to the traditional drape coefficient method, the DC of each of the fabrics was determined according to the British Standard for the Assessment of Drape of Fabrics (BS 5058). As well, the 3-D scanning drape results compared to the two-dimensional stiffness results, the ASTM Test Method for the Stiffness of Fabrics using the cantilever bending approach was also used (ASTM D1388). Four specimens $25 \mathrm{~mm}$ by $200 \mathrm{~mm}$ were prepared for both the warp direction and for the weft direction. Specimens were conditioned in the standard atmosphere of $20^{\circ} \mathrm{C} \pm 2{ }^{\circ} \mathrm{C}$ and at $65 \% \pm 2 \%$ relative humidity for a minimum of 24 hours prior to testing. 


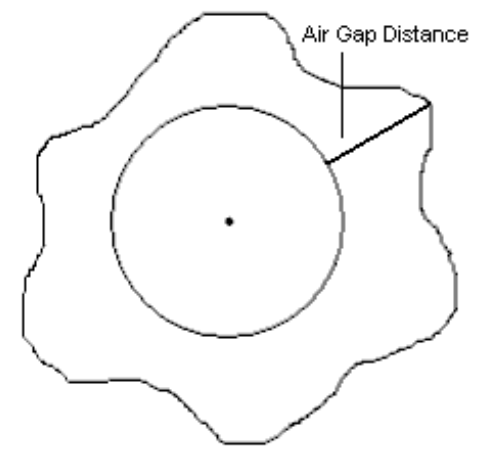

Fig. 1. Air gap distance.

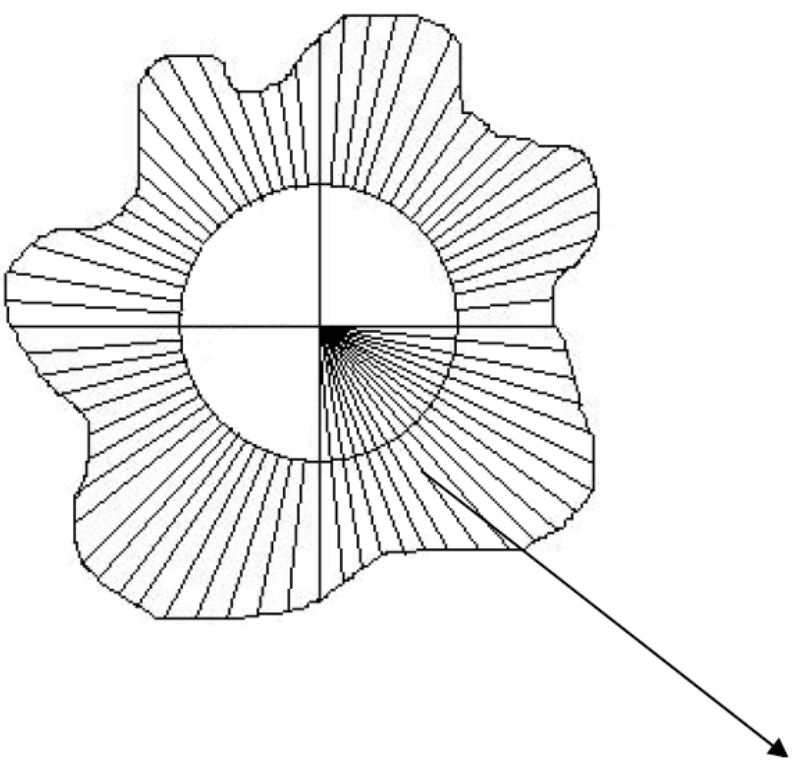

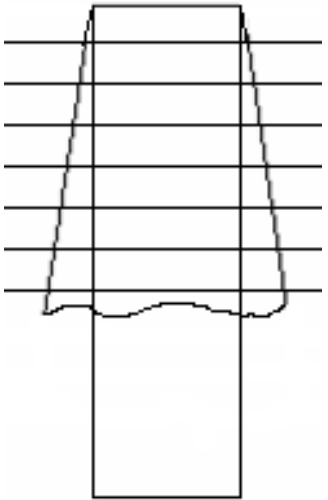

Fig. 2. Horizontal planes for cross-sections at different elevations.

$5^{\circ}$ intervals

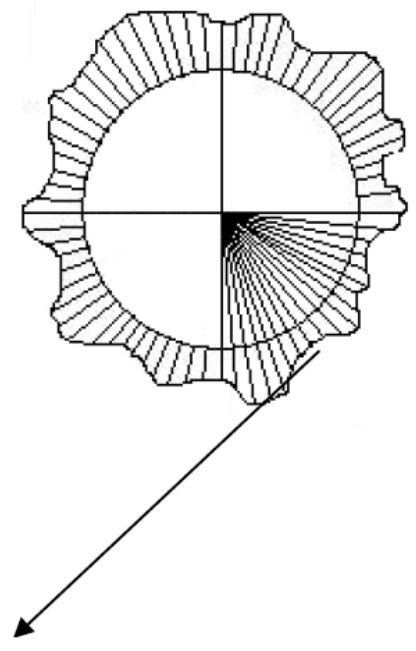

Fig. 3. Differences in drape shapes and air gap distances between a less drapeable fabric (left) and a more drapeable fabric (right).

A fabric with lower drape will exhibit a higher average air gap distance, whereas a fabric with higher drape will exhibit a lower average air gap distance. Thus, an inverse relationship between the average air gap distance and the amount of drape is anticipated. If a fabric has $100 \%$ drape, the total air gap distance would have a theoretical minimum of $0 \mathrm{~cm}$. Considering the air gap information along the entire length of a draped fabric via cross-sections (Figure 2) will provide more information about the appearance of a draped fabric than the DC.

\section{Test apparatus and procedure}

Three-dimensional scanning was conducted using the Vitus 3-D whole body scanner by Human Solutions. The scanner is connected to a computer equipped with the ScanWorX and Anthroscan 3-D measurement software. A cylinder $120 \mathrm{~cm}$ tall and $22 \mathrm{~cm}$ in diameter was constructed for the fabrics to be draped over. This diameter was thought to be a better representation of the size of a human torso than those of a smaller diameter used in other methods. This size would also prove useful in future research where the drape of garments over a human form would be determined. A pin was affixed to the center of the top of the cylinder to allow the central placement of the test specimen. An $18 \mathrm{~cm}$ diameter drapemeter as per the requirements outlined in BS 5058 was constructed for the measurement of the DC. 


\section{Results and discussion}

The images of the 3-D scanning are presented visually in Figure 4. The fabrics on the right were found to have higher average air gap distances and hence had lower degrees of drape. Fabrics on the left had lower average air gap distances and hence higher degrees of drape. It is more evident from the top views in Figure 4 than from the side views that as the fabric drape increases, the overall average air gap sizes between the cylinder and the outside edge of the fabric in general decreases.

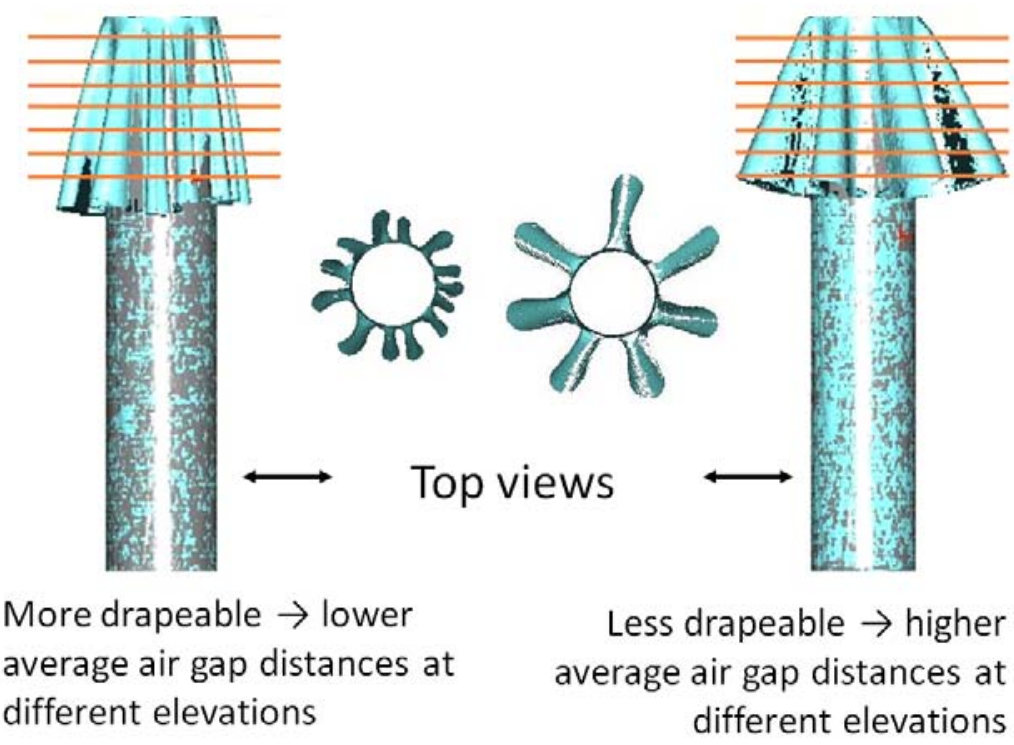

Fig. 4. Scanned image of draped fabric

Table 1 provides a side-by-side comparison of the drape rankings produced by the 3-D scanner, drapemeter, and cantilever stiffness approaches for the measurement of drape. Each method showed that fabric $G$ was the most drapeable fabric and that fabric $F$ was the next most drapeable. Each method showed that the Fabric $A$ was the least drapeable. However, the rankings for the Fabric $B, C$, $D$ and $E$ across the different approaches were inconsistent, as the relative drape of these fabrics varied across the tests. One of the reasons that contribute the difference in rankings for some is likely because of the different cylinder to fabric diameter ratios used in the two tests. In the drapemeter test, the ratio of the cylinder to fabric diameter was $18 \mathrm{~cm}$ to $36 \mathrm{~cm}$ whereas in the 3-D scanning, the ratio of the cylinder to fabric diameter was $22 \mathrm{~cm}$ to $100 \mathrm{~cm}$. There was significantly more fabric overhang in the 3-D scanning approach, likely accounting for the differences in drape rankings. Explaining why the fabrics exhibit the differing amounts of drape that they do is difficult due to the complex set of factors that influence drape, as mentioned in the literature review. At this early stage of the research though, the physical and mechanical properties that impact fabric drape have not been investigated, but will be an area for future work.

Table 1 Comparison of relative drape rankings for different drape tests.

\begin{tabular}{|c|c|c|c|c|c|c|c|}
\hline & \multicolumn{3}{|c|}{ Least drape } & \multicolumn{2}{|c|}{$\Longleftrightarrow$} & \multicolumn{2}{|c|}{ Most drape } \\
\hline Fabric drape sequence & $A$ & B & $\mathrm{C}$ & $\mathrm{D}$ & $E$ & $\mathrm{~F}$ & G \\
\hline $\begin{array}{l}\text { Drape measurement } \\
\text { (BS 5058), \% }\end{array}$ & 84.0 & 73.3 & 71.7 & 59 & 58.7 & 49.5 & 39.3 \\
\hline Fabric drape sequence & $A$ & $\mathrm{C}$ & $B$ & $E$ & $\mathrm{D}$ & $\mathrm{F}$ & $\mathrm{G}$ \\
\hline $\begin{array}{l}\text { Drape measurement } \\
\text { (ASTM D 1388), mm }\end{array}$ & 5.0 & 4.0 & 3.8 & 3.4 & 2.7 & 2.4 & 1.7 \\
\hline Fabric drape sequence & A & $E$ & $\mathrm{C}$ & $B$ & $\mathrm{D}$ & $\mathrm{F}$ & G \\
\hline $\begin{array}{c}\text { Drape measurement - } \\
\text { 3D body scanning/air } \\
\text { gap size }\end{array}$ & 3472 & 2820 & 2508 & 2339 & 2100 & 1631 & 1187 \\
\hline
\end{tabular}




\section{Conclusions}

The objectives of this research were to determine whether 3-D scanning technology could be used to measure the drape of fabrics, whether 3-D scanning could differentiate between the drape of different fabrics, whether 3-D scanning was an improvement over conventional drape measurement techniques, and specifically, whether air gap information obtained from 3-D scanning could be used as a measure of fabric drape. From the results obtained, the following conclusions can be made: 1) 3-D scanning is able to measure the drape of fabrics; 2) 3-D scanning can differentiate between the differing amounts of drape exhibited by fabrics, 3) 3-D scanning is an improvement over traditional drape measurement techniques as additional information about an entire draped form, for example, the appearance, can be obtained, 4) Air gap distances obtained from 3-D scanning is capable of differentiating between fabrics with low drape and fabrics with high drape, and provides more information about the appearance of a draped form than the DC.

\section{References}

1. Cusick, G. E. (1965): "The dependence of fabric drape on bending and shear stiffness", Journal of the Textile Institute, 56, T596-T606.

2. Stump, D. M., \& Fraser, W. B. (1996): "A simplified model of fabric drape based on ring theory", Textile Research Journal, 66(8), 506-514.

3. Brand, R. H. (1964): "Measurement of fabric aesthetics: Analysis of aesthetic components", Textile Research Journal, 34, 791-804.

4. Chu, C. C., Cummings, C. L., \& Teixeira, N. A. (1950): "Mechanics of elastic performance of textile materials part $\mathrm{V}$ : A study of the factors affecting the drape of fabrics - the development of a drape meter", Textile Research Journal, 20, 539-548.

5. Chu, C. C., Platt, M. M., Hamburger, W. J. (1960): "Investigation of the factors affecting the drapeability of fabrics", Textile Research Journal, 30(1), 66-67.

6. Saville, B. P. (1999): "Physical testing of textiles", Cambridge, England: Woodhead Publishing Limited.

7. Govindaraj, M., \& Vallamshettla, A. (1999): "Physically based fabric drape models for computer-aided design", National Textile Center Annual Report: 198-P02.

8. Collier, B. J., \& Epps, H. H. (1999): "Fabric hand and drape", In B. J. Collier, \& H. H. Epps (Eds.), Textile testing and analysis (259-280). New Jersey: Prentice Hall.

9. Rödel, H., Ulbricht, V., Krzywinski, S., Schenk, A., \& Fischer, P. (1998): "Simulation of drape behaviour of fabrics", International Journal of Clothing Science and Technology, 10(3/4), 201-208.

10. Collier, B. J. (1991): "Measurement of fabric drape and its relation to fabric mechanical properties and subjective evaluation", Clothing and Textiles Research Journal, (10)1, 46-52.

11. Postle, R. \& Postle, J. R. (1998). The dynamics of fabric drape. International Journal of Clothing Science and Technology, 10(3/4), 305-312.

12. Collier, B. J. (1990). Assessment of fabric drape. The FIT Review, 6(2), 40-43.

13. Stylios, G. K., \& Wan, T. R. (1999): "The concept of virtual measurement: 3D fabric drapeability", International Journal of Clothing Science and Technology, 11(1), 10-18.

14. Hatch, K. (1993): "Textile science", Minneapolis: West Publishing.

15. Kenkare, N. \& May-Plumlee. (2005): "Evaluation of drape characteristics in fabrics", International Journal of Clothing Science and Technology, 17(2), 109-123. 